\section{KIR3DL2 : une nouvelle étape dans l'histoire du syndrome de Sézary}

Nicolas Ortonne, Martine Bagot, Armand Bensussan

$>$ Le syndrome de Sézary (SS) fait partie des lymphomes T cutanés primitifs. II est caractérisé par la prolifération d'un

clone lymphocytaire T mature, de phénotype mémoire $\mathrm{CD} 45 \mathrm{RO}^{+} \mathrm{CD}_{4}^{+}$qui se localise dans le sang et la peau, avec une

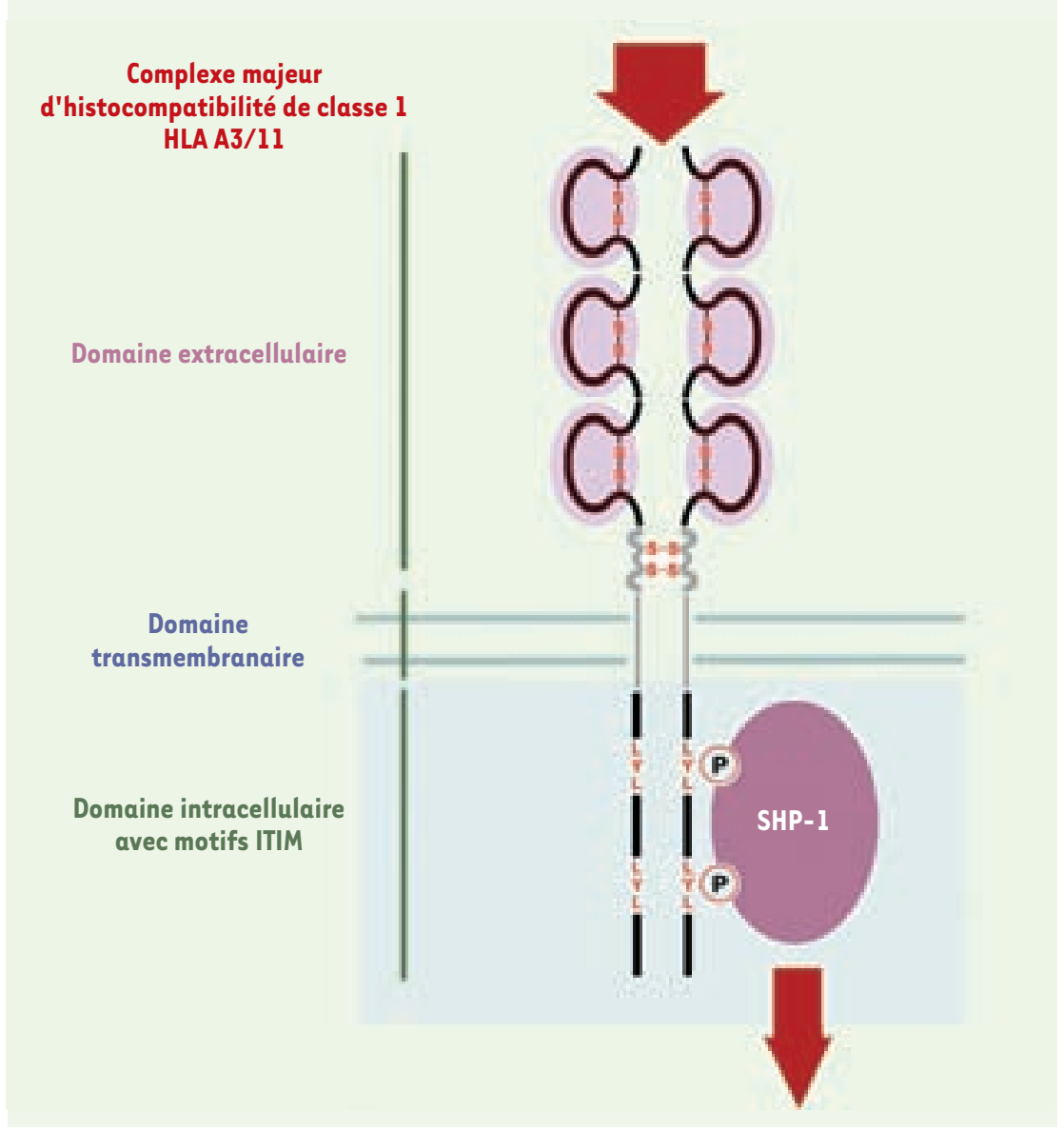

Figure 1. Structure de KIR3DL2. KIR3DL2/CD158k/pl40 est un homodimère de p70. Chaque monomère est constitué d'une partie extracellulaire faite de trois domaines immunoglobuliniques, d'une partie transmembranaire et d'une partie longue intracytoplasmique. Concernant les lymphocytes NK, la transduction du signal implique une cascade d'événements qui ont été bien décrits. Après engagement avec son ligand physiologique (les molécules du complexe majeur d'histocompatibilité de classe I), les résidus tyrosine portés par les motifs ITIM de la portion intracellulaire du récepteur sont phosphorylés et recrutent la molécule phosphatase cytosolique SHP-1 qui va permettre la déphosphorylation des tyrosine kinases recrutées lors de l'engagement des récepteurs activateurs.
N. Ortonne : Inserm U659,

Faculté de Médecine de Créteil,

8, rue du Général Sarrail, 94000 Créteil

et Département de Pathologie,

Hopital Henri Mondor,

51, avenue du Maréchal de Lattre de Tassigny, 94010 Créteil, France.

M. Bagot, A. Bensussan : Inserm U659,

Faculté de Médecine de Créteil,

8, rue du Général Sarrail, 94000 Créteil

et Service de Dermatologie,

Hôpital Henri Mondor,

51, avenue du Maréchal de Lattre de Tassigny, 94010 Créteil, France.

Armand.Bensussan@creteil.inserm.fr

atteinte généralement diffuse, se traduisant cliniquement par une érythrodermie. Il se distingue par son mauvais pronostic, puisque la survie médiane des patients est d'environ 5 ans [1]. Le diagnostic de SS est un problème en pratique médicale, car de nombreuses autres maladies dermatologiques bénignes peuvent se présenter sous la forme d'une érythrodermie. En l'absence de marqueur spécifique, la caractérisation des cellules malignes dans le sang et la peau se fait de façon approximative, par leurs caractéristiques morphologiques et par la détection d'un clone lymphocytaire T. On sait que l'aspect morphologique des cellules de Sézary, au noyau irrégulier «cérébriforme » n'est pas spécifique des cellules malignes. S'agissant de la détection de clones lymphocytaires $\mathrm{T}$, on peut en trouver dans de nombreuses situations, y compris certaines dermatoses inflammatoires bénignes, au cours desquelles peut se produire une expansion clonale de lymphocytes $T$ réactionnels. C'est certainement grâce à la composante leucémique, permettant la culture à long terme de lignées de cellules tumorales circulantes [2], que les plus grandes avancées dans le domaine des lymphomes T cutanés ont été réalisées dans le SS. C'est notamment par cette approche que le récep- 
teur KIR3DL2/CD158k a été initialement montré comme le premier marqueur de surface des cellules de Sézary [3-5].

\section{Le récepteur KIR3DL2}

Les KIR (killer immunoglobulin-like receptors) sont des récepteurs des lymphocytes tueurs naturels (NK), dont les gènes sont localisés sur le chromosome 19 en q13.4; ils interagissent avec les molécules $d u$ complexe majeur d'histocompatibilité de classe I et transduisent des signaux activateurs ou inhibiteurs des fonctions cellulaires effectrices et immunorégulatrices. Ils participent à la régulation de la tolérance au soi par le système immunitaire non adaptatif. La nomenclature habituellement utilisée pour les désigner témoigne de la structure de chaque récepteur, en indiquant le nombre de domaine immu- noglobulinique extracellulaire ainsi que l'existence d'une partie longue ( $L$ ) ou courte (S) intracytoplasmique responsable d'une signalisation inhibitrice ou activatrice, respectivement. Ainsi, le récepteur KIR3DL2 possède trois domaines extracellulaires et joue un rôle inhibiteur, grâce aux motifs ITIM (immunoreceptor tyrosine-based inhibitory motifs) contenus dans sa partie longue intracytoplasmique (Figure 1). Outre certains lymphocytes NK, le récepteur KIR3DL2/CD158k/p140 est exprimé par des populations très minoritaires de lymphocytes CD8 circulants.

\section{KIR3DL2/CD158k, clé de voûte}

du diagnostic de syndrome de Sézary

C'est en étudiant les clones T CD4 ${ }^{+}$circulants de malades atteints de SS que nous avons pu montrer que KIR3DL2 carac-

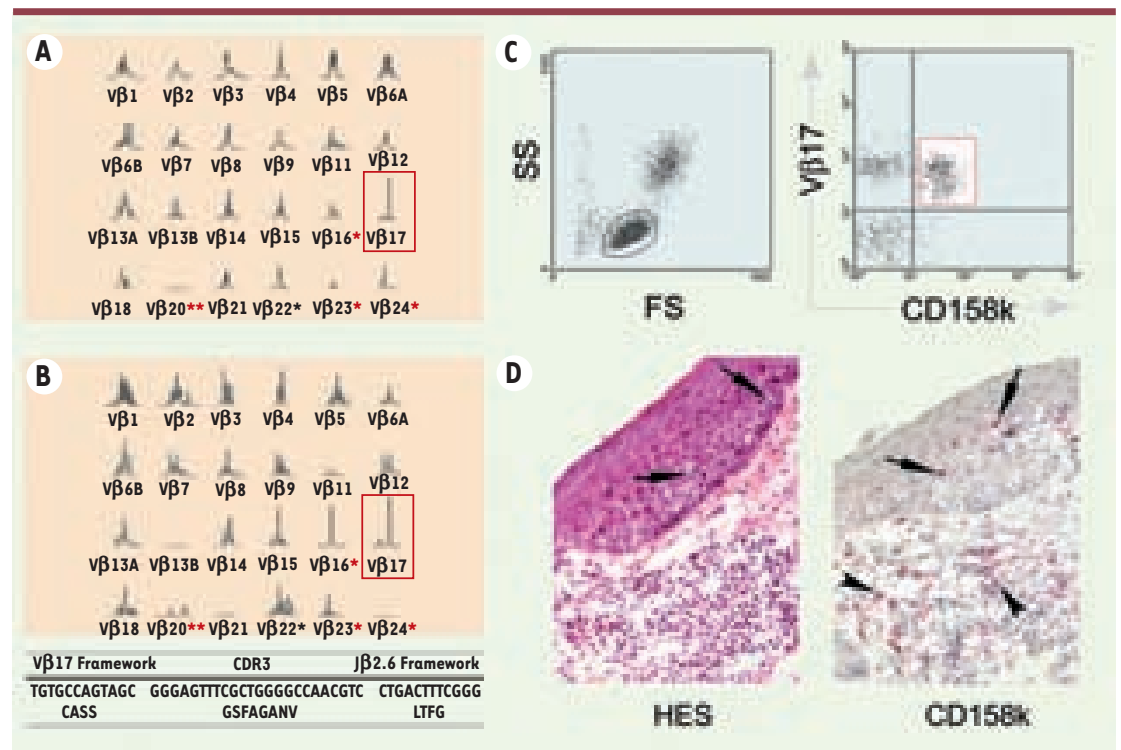

Figure 2. Caractérisation des cellules malignes chez un malade ayant un syndrome de Sézary débutant. A. L'analyse de taille des produits d'amplification $V \beta-C \beta$, première étape de l'étude de clonalité par la technique de l'immunoscope, montre la présence dans le sang d'un clone lymphocytaire $T$ caractérisé par un segment $V \beta 17$. Cette analyse révèle par ailleurs des anomalies du répertoire $T$ des lymphocytes circulants, avec perte de la famille $V \beta 20^{* *}$ et des profils restreints*. B. Le même clone lymphocytaire T est trouvé dans la peau érythrodermique, associé à d'autres clones (familles $V \beta 15$ et $V \beta 16)$. La séquence du produit TCRV $\beta 17-C \beta$ est identique dans l'échantillon de sang et de peau, confirmant la localisation cutanée et sanguine de ce clone. $C$. Dans le sang, l'analyse par cytométrie en flux en double marquage montre que la population TCRV $\beta 17$ exprime le marqueur CD158k/KIR3DL2, confirmant sa nature tumorale. $D$. Dans la peau érythrodermique, on trouve des lymphocytes exprimant en surface KIR3DL2, à la fois dans l'épiderme (flèches) et le derme (pointes de flèches), associés à d'autres lymphocytes non tumoraux, en accord avec la mise en évidence par l'immunoscope d'un répertoire T varié associé au clone tumoral V $\beta 17$. térise les cellules malignes du SS, et que des clones $T$ non tumoraux circulent également dans le sang des malades [6]. L'analyse des réarrangements $\gamma$ à l'échelle génomique s'est imposée comme technique de référence pour la détection des clones lymphocytaires $T$ en pratique de routine [7]. Elle peut en effet être appliquée à tout échantillon contenant de l'ADN et nécessite un nombre limité d'amorces, du fait de l'organisation génique relativement simple des gènes du TCR $\gamma$. La technique de l'immunoscope, reposant sur l'analyse moléculaire de la région CDR3 hypervariable des transcrits de la chaîne $\beta$ du TCR [8], permet de caractériser plus précisément les différents clones lymphocytaires T présents dans un échantillon. C'est cette technique que nous avons utilisée pour analyser le répertoire lymphocytaire T de malades avec un SS. Outre des pertes ou une restriction au sein de certaines familles de gènes $V$ du TCR $\beta$, qui traduisent un état d'immunosuppression avancé bien connu chez ces malades, nous avons pu ainsi identifier dans le sang un ou plusieurs clones. Pour déterminer leur nature néoplasique ou réactionnelle, nous avons apprécié au sein de ces clones l'expression de KIR3DL2 et avons établi des corrélations avec l'expression clinique de la maladie. Pour la plupart des malades, nous avons ainsi détecté le clone tumoral, par son phénotype CD158k/KIR3DL2+ (Figure 2A), et montré que celui-ci devient indétectable chez des patients en rémission complète après traitement. Le clone ainsi caractérisé dans le sang peut être retrouvé dans la peau (Figure 2B), où I'on retrouve des lymphocytes exprimant KIR3DL2 (Figure 2B), en accord avec la double expression sanguine et cutanée de la maladie. De façon intéressante, par cette approche, nous avons également mis en évidence, en plus de la population tumorale, d'autres populations lymphocytaires T clonales. Pour certaines d'entre elles, nous montrons leur caractère non malin, par l'absence d'expression de KIR3DL2 et leur persistance au stade de 
rémission complète clinique. Ces résultats indiquent clairement que les études moléculaires de clonalité ne permettent pas de caractériser spécifiquement les cellules tumorales, ce que rend possible le marqueur KIR3DL2. II est toutefois important de signaler que la caractérisation d'un clone $T$ identique dans le sang et la peau reste certainement un bon argument en faveur du diagnostic de SS en situation d'érythrodermie [7]. Au-delà de son intérêt comme marqueur diagnostique, la mise en évidence de l'expression de KIR3DL2 à la surface des lymphocytes T CD4 $4^{+}$malins du SS suscite de nombreuses interrogations, notamment concernant sa fonction et plus particulièrement son rôle dans la prolifération et la résistance à l'apoptose des cellules tumorales. Par ailleurs, les lymphocytes T CD4 $4^{+}$normaux n'expriment par KIR3DL2, et l'on peut se demander si les voies de signalisation moléculaires impliquées par l'engagement de ce récepteur dans les lymphocytes T tumoraux du SS sont les mêmes que dans les lymphocytes NK.
Un nouvel espoir

pour le traitement des malades?

Devant la difficulté d'obtenir des rémissions complètes à long terme chez les malades atteints du SS, la mise au point de nouvelles armes thérapeutiques s'avère nécessaire. L'utilisation d'anticorps monoclonaux humanisés ciblant des antigènes de différenciation lymphocytaire de surface a permis ces dernières années de réaliser des progrès significatifs dans le traitement des lymphomes systémiques. Il est maintenant logique de penser que KIR3DL2 pourrait représenter une bonne cible d'immunothérapie dans le SS. Et cette idée paraît d'autant plus séduisante que les cellules de Sézary sont bien sensibles à la lyse induite par les lymphocytes NK in vitro et que celle-ci peut être potentialisée par l'utilisation d'anticorps monoclonaux anti-KIR3DL2 [9]. $\diamond$

KIR3DL2: a new step for the management of patients with Sezary syndrome

\section{RÉFÉRENCES}

1. Foulc P, N'Guyen JM, Dreno B. Pronostic factors in Sezary syndrome: a study of 28 patients. Br J Dermatol $2003 ; 149$ : 1152-8.
2. Poszepczynska $\varepsilon$, Bagot $M$, Echchakir $H$, et al. Functional characterization of an IL-7-dependent $\mathrm{CD}^{+} \mathrm{CD} 8$ alphaalpha ${ }^{+}$Th3-type malignant cell line derived from a patient with a cutaneous T-cell lymphoma. Blood 2000 ; 96 : 1056-63.

3. Bagot M, Moretta A, Sivori S, et al. $\mathrm{CD}^{+}$cutaneous T-cell lymphoma cells express the p140-killer cell immunoglobulin-like receptor. Blood 2001; $97: 1388-91$

5. Poszepczynska-Guigne $\varepsilon$, Schiavon V, D'Incan M, et al. CD158k/KIR3DL2 is a new phenotypic marker of Sezary cells: relevance for the diagnosis and follow-up of Sezary syndrome. J Invest Dermatol 2004 . $122: 820-3$.

4. Wechsler J, Bagot M, Nikolova M, et al. Killer cell immunoglobulin-like receptor expression delineates in situ Sezary syndrome lymphocytes. J Pathol 2003; 199: 77-83.

6. Ortonne N, Gaudez C, Huet D, et al. Significance of circulating T cells clones in Sezary syndrome. Blood $2006 ; 107: 4030-8$.

7. Delfau-Larue MH, Laroche L, Wechsler J, et al. Diagnostic value of dominant $\mathrm{T}$-cell clones in peripheral blood in 363 patients presenting consecutively with a clinical suspicion of cutaneous lymphoma. Blood 2000; $96: 2987-92$.

8. Pannetier C, Levraud JP, Lim A, et al. The immunoscope technique for analysis of TCR repertoire. In: Oksenberg $J R$, ed. The human antigen $T$ cell receptor, selected protocols and applications. London: Chapman and Hall, 1998 : 287-325.

9. Bouaziz JD, Ortonne N, Giustiniani J, et al. Circulating natural killer lymphocytes are potential cytotoxic effectors against autologous malignant cells in Sezary syndrome patients. J Invest Dermatol 2005; 125 : 1273-8.

\section{NOUVELLE}

\section{Wnt et cellules souches intestinales: des liaisons dangereuses...}

Pauline Andreu, Christine Perret, Béatrice Romagnolo
Inserm U567, CNRS UMR 8104, Université Paris V, Institut Cochin, 24, rue du Faubourg Saint-Jacques, 75014 Paris, France. romagnolo@cochin.inserm.fr
> La notion de cellules souches cancéreuses date des années 1950 mais pendant longtemps les scientifiques n'ont pas été en mesure de prouver leur existence. Récemment, différents travaux ont permis d'apporter des rapports de causalité entre cellules souches et cellules cancéreuses [1]. En effet les cellules cancéreuses partagent un grand nombre de similarités avec les cellules cancéreuses. II devient donc urgent de mieux comprendre ces cellules souches qui ne nous veulent pas que du bien... Mieux comprendre une cellule souche, c'est une étape probablement clé pour l'étude des mécanismes impliqués dans l'initiation du processus tumoral. L'épithélium intestinal est un exemple frappant de cette corrélation entre cellules souches et cellules cancéreuses [2].

Les cellules de l'épithélium intestinal sont régénérées tout au long de la vie. Dès la naissance se constitue le compartiment cryptique dans lequel résident les cellules prolifératives, cellules souches et progéniteurs (Figure 1). Suite à une division asymétrique, une cellule souche donne naissance à une nouvelle cellule souche et à un progéniteur destiné à se différencier. Il est bien établi que les cellules souches intestinales donnent naissance aux quatre types de cellules épithéliales différenciées: cellules neuroendocrines, cellules à mucus, entérocytes, et cellules de Paneth. L'homéostasie de l'épithélium intestinal est donc assurée grâce à un équilibre entre la production des cellules différenciées, leur migration dans la villosité vers la 